\title{
Réponse des nématodes à la perturbation des forêts dans la région d'Oumé, Côte d'Ivoire.
}

\author{
Jacob NandJuI ${ }^{1 *}$, Philippe G. GnonhourI ${ }^{2}$, Jérôme E. TondoH ${ }^{3} \&$ Yao TANO $^{4}$, \\ ${ }^{1}$ Laboratoire de Zoologie agricole / Ecole Supérieure d'Agronomie de Yamoussoukro BP 1313 Yamoussoukro (Côte d'Ivoire) \\ ${ }^{2}$ Centre National de Recherches Agronomiques 01 BP 1740 Abidjan 01(Côte d'Ivoire) \\ ${ }^{3}$ UFR Sciences de la Nature / Université d'Abobo-Adjamé 02 BP 801 Abidjan 02 (Côte d'Ivoire) \\ ${ }^{4}$ UFR Biosciences / Université de Cocody 22 BP 582 Abidjan 22 (Côte d'Ivoire) \\ *Auteur pour les correspondances (E-mail : jacobnandjui@yahoo.fr) \\ Reçu le 11-05-2006, accepté le 13-11-2006.
}

\section{Résumé}

La sensibilité des nématodes aux perturbations des forêts a été étudiée à l'échelle du paysage dans la région d'Oumé. L'échantillonnage a été réalisé le long d'un gradient de perturbations du milieu, partant des forêts vers le domaine rural, en raison de six répétitions par habitat. Les résultats ont montré que les nématodes libres représentent $68 \%$ du peuplement de nématodes. Neuf genres de nématodes phytoparasites (Criconemella, Helicotylenchus, Heterodera, Hoplolaimus, Meloidogyne, Pratylenchus, Radopholus, Rotylenchulus et Xiphinema) ont été identifiés. Parmi ces nématodes, Rotylenchulus sp et Xiphinema sp peuvent être considérés comme bioindicateurs à cause de la tendance à la réduction de leurs populations le long du gradient d'utilisation de sol. ( $r=-0,74 ; P=0,03$ et $r=-0,77 ; P=0,03$ ).

Mots clés : bioindicateur, diversité, indice d'utilisation de sol, nématodes, paysage.

\section{Abstract}

\section{Response of nematodes to forest perturbations in the Oumé region, Côte d'Ivoire}

The sensitivity of nematode communities to forest perturbations has been studied in the region of Oumé at the landscape level. Six samples were collected along a gradient of perturbations from forests to lands crops areas. The results showed that the free-living nematodes accounted for $68 \%$ of the nematode community. The plant-parasitic nematode community was composed by nine genera (Criconemella, Helicotylenchus, Heterodera, Hoplolaimus, Meloidogyne, Pratylenchus, Radopholus, Rotylenchulus and Xiphinema). Rotylenchulus sp and Xiphinema sp are likely to be considered as bio-indicators of forest degradation as their populations are significantly reduced along the gradient of land use change $(r=-0.74, P=0.03$ and $r=-0.77, P=0.03)$.

Key words: bio-indicator, diversity, land use index, landscape, nematodes.

\section{Introduction}

Certains groupes zoologiques ont une forte sensibilité liée au degré de perturbation de leurs habitats. C'est le cas des nématodes qui représentent à la fois un groupe biologique homogène, très abondant et très diversifié avec plus de 26000 espèces décrites (Bongers, 1990).
Dans le sol, les nématodes vivent sous forme de communautés soumises à des interactions avec les autres facteurs biotiques et abiotiques (Yeates, 1994). Ces interactions sont à l'origine de la composition des peuplements observés (Lal, 1991; Gupta, 1994). Les indices calculés à partir de la structure des peuplements de nématodes peuvent être utilisés pour évaluer les 
perturbations écologiques du sol (Solhenius et al., 1987; Wardle et al., 1995). Ainsi, les nématodes constituent des modèles biologiques pour étudier l'impact des changements de pratiques agricoles à l'échelle du paysage (Freckman \& Ettema, 1993).

En Côte d'Ivoire, les investigations menées sur de nombreuses plantes (Luc \& De Guiran, 1960), à l'échelle de la parcelle dans la forêt de Taï (Fortuner \& Couturier, 1983) et dans des jachères de la région d'Oumé (Gnonhouri \& Adiko, 2000) ont révélé que certaines espèces se rencontrent à la fois dans la forêt et dans les milieux cultivés, alors que d'autres sont inféodés soit aux écosystèmes naturels, soit aux milieux anthropisés. Très peu d'études ont été menées sur les peuplements de nématodes phytoparasites à l'échelle du paysage; échelle privilégiée d'étude des modifications de la biodiversité par les activités humaines (Halffter, 1998).

L'objectif de cette étude est de mesurer l'impact des activités humaines sur l'abondance et la diversité des nématodes à l'échelle d'un paysage couvrant différents écosystèmes. Elle repose sur l'hypothèse selon laquelle les peuplements de nématodes peuvent être utilisés comme indicateurs de perturbation de forêts (Ettema \& Bongers, 1993 ; Freckman \& Ettema, 1993 ; Neher \& Campbell, 1994).

\section{Matériel et méthodes}

\subsection{Site d'étude et types d'habitats}

Le site d'étude est localisé dans la région d'Oumé, Centre Ouest de la Côte d'Ivoire $\left(5^{\circ} 30\right.$ Ouest et $6^{\circ} 31$ Nord). Le sol, de type ferrallitique, est distribué de façon homogène à l'échelle du paysage ( $P$. Angui, Université d'Abobo-Adjamé, Abidjan, Côte d'Ivoire, communication personnelle). Le climat est chaud et humide. Les températures mensuelles, comprises entre $24^{\circ} 5$ et $27^{\circ} 2 \mathrm{C}$, présentent de faibles variations. La pluviométrie annuelle était d'environ $1541 \mathrm{~mm}$, répartie selon un régime bimodal à deux saisons de pluies (mai à juin; septembre à octobre) et deux saisons sèches (novembre à février ; juillet à août) (SODEXAM, Gagnoa, Côte d'Ivoire, résultats non publiées). Le site couvre 400 ha et comporte plusieurs habitats, dont la composition floristique a été décrite par N'Guessan et al. (CNF,
Abidjan, Côte d'Ivoire, résultats non publiés). Huit habitats ont fait l'objet de notre étude :

- une forêt primaire semi décidue dans laquelle l'action de l'homme est très faible et se limite à des pistes réalisées à l'intérieur de la parcelle. Le couvert est très dense et le sous-bois est moyennement dégagé avec des lianes et des bois morts ;

- une forêt secondaire qui a subi un incendie en 1983 et qui depuis lors est restée protégée. Le couvert est dense avec un sous-bois riche en lianes, en bois morts, en arbustes et une forte présence de Chromolaena odorata (Asteraceae) ;

- des reboisements plurispécifiques qui sont des parcelles plantées avec Gmelina arborea et Tectona grandis (Verbenaceae), Cedrela toona (Meliaceae), Temrinalia ivorensis et $T$. superba (Combretaceae). On note une forte présence de Chromolaena odorata ;

- une plantation de Teck de dix ans, mise en place en 1994. Le couvert est assez important avec un sous-bois dégagé et une litière riche en feuilles mortes;

- une plantation de Teck de quatre ans, mise en place en 2000 . Le couvert végétal est faible ;

- des plantations de cacaoyers localisées à la périphérie de la forêt. Les relevés floristiques réalisés donnent quelques espèces dominantes: Albizia adianthifolia (Mimosaceae), Axonopus compressus (Poaceae), Chromolaena odorata (Asteraceae), Elaeis guineensis (Arecaceae);

- des zones à cultures vivrières. Ce sont des parcelles de moins d'un hectare dominées par Chromolaena odorata (Asteraceae), Trichilia monadelpha (Meliaceae), Theobroma cacao (Sterculiaceae), Sterculia tragacantha (Sterculiaceae), Solanum torvum (Solanaceae), Panicum laxum (Poaceae) ;

- des jachères de deux à cinq ans qui sont des milieux forestiers dégradés par la pratique de l'agriculture et remplacés par des végétations secondaires.

Un indice d'utilisation de sol (IUS), permettant de déterminer le degré de perturbation de chaque habitat, a été calculé (Tableau 1) selon la méthode 
suivante :

IUS $=(D+F+I+P) / N$ (N'Doumé et al., 2005) ; où $D$ : durée d'exploitation du sol au moment du calcul de l'indice, $F$ : fréquence de mise à feu (nombre de mise à feu par an), I: fréquence d'utilisation d'intrant inorganique, $P$ : fréquence d'utilisation de pesticide, $\mathrm{N}$ : le nombre de facteurs utilisés dans le calcul de l'indice.

Tableau 1: Fréquences des populations de nématodes phytoparasites par habitat.

\begin{tabular}{lcccccccc}
\cline { 2 - 8 } \multicolumn{1}{c}{ Fréquences (f) } & $\mathrm{Fp}$ & $\mathrm{Fs}$ & $\mathrm{Rps}$ & $\mathrm{Tk10}$ & $\mathrm{Ca}$ & $\mathrm{Tk} 4$ & $\mathrm{~J}$ & $\mathrm{cv}$ \\
\cline { 2 - 8 } & $(0)$ & $(0,13)$ & $(0,26)$ & $(0,27)$ & $(0,28)$ & $(0,31)$ & $(0,31)$ & $(0,38)$ \\
\hline Meloidogyne sp & 0,5 & 0,7 & 0,8 & 0,8 & 0,7 & 0,7 & 1,0 & 1,0 \\
Helicotylenchus sp & 0,5 & 0,5 & 0,5 & 0,3 & 0,5 & 0,0 & 0,2 & 0,3 \\
Rotylenchulus sp & 0,8 & 0,8 & 0,3 & 0,0 & 0,5 & 0,5 & 0,5 & 0,0 \\
Xiphinema sp & 0,5 & 0,5 & 0,5 & 0,2 & 0,2 & 0,0 & 0,0 & 0,0 \\
Heterodera sp & 0,0 & 0,2 & 0,0 & 0,3 & 0,3 & 0,0 & 0,2 & 0,2 \\
Pratylenchus sp & 0,0 & 0,2 & 0,0 & 0,2 & 0,0 & 0,3 & 0,2 & 0,0 \\
Hoplolaimus sp & 0,0 & 0,0 & 0,2 & 0,0 & 0,0 & 0,0 & 0,0 & 0,0 \\
Criconemella sp & 0,0 & 0,0 & 0,2 & 0,0 & 0,0 & 0,0 & 0,0 & 0,0 \\
Radopholus sp & 0,0 & 0,0 & 0,0 & 0,2 & 0,0 & 0,0 & 0,0 & 0,0 \\
\hline
\end{tabular}

Fp : forêt primaire ; Fs : forêt secondaire ; Rps : reboisements plurispécifiques ; Tk10 : plantations de teck de 10 ans ; Ca : plantations de cacaoyers ; Tk4 : plantations de teck de quatre ans ; $J$ : jachères et $\mathrm{Cv}$ : milieux de cultures vivrières. Les valeurs entre parenthèses représentent les indices d'utilisation du milieu

\section{.2.2. Stratégie d'échantillonnage}

L'échantillonnage a consisté à réaliser des prélèvements de sol pendant la petite saison sèche (juillet à août 2004). Les points échantillonnés font partie d'une grille d'échantillonnage mise au point par le Projet Conservation et Gestion Durable de la Diversité Biologique des sols (CSM-BGBD). Cette grille comporte 107 points dont 100 sont équidistants de $200 \mathrm{~m}$. Les sept autres points distants de 100 $\mathrm{m}$ ont été rajoutés afin de corriger la sous représentativité des plantations de cacaoyers et des cultures vivrières. Dans chaque habitat, six points ont été échantillonnés de manière aléatoire. Les échantillons ont été collectés à 20

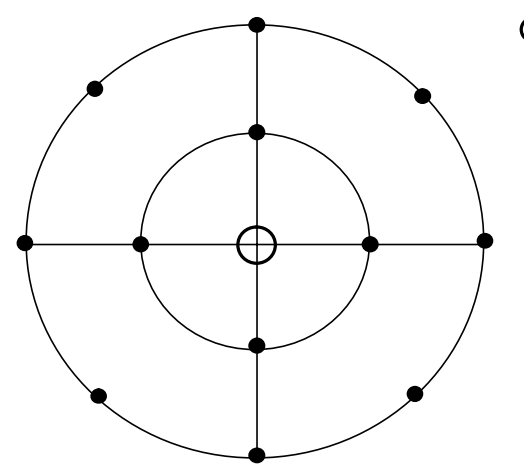

Figure 1: Schéma du dispositif de prélèvement des échantillons de sol
Point d'échantillonnage sur la grille

Point de prélèvement de sol

Petit rayon $: 3 \mathrm{~m}$

Grand rayon : $6 \mathrm{~m}$

$\mathrm{cm}$ de profondeur. Chaque échantillon résulte de 12 prélèvements élémentaires répartis selon un schéma bien précis (Fig. 1). Autour de chaque point d'échantillonnage, un cercle de $3 \mathrm{~m}$ a été délimité autour de deux lignes perpendiculaires. Les points d'intersection de ce cercle avec les lignes représentent les quatre premiers points de prélèvement. Les autres points sont répartis sur un cercle de $6 \mathrm{~m}$ de rayon concentrique au premier. Les cercles ont été délimités à partir de cordes de $3 \mathrm{~m}$ et de $6 \mathrm{~m}$. Après prélèvement, les 12 carottes de terre ont été mélangées et débarrassées des grosses particules pour donner un échantillon composite de sol de 1 à $1,5 \mathrm{~kg}$. Chaque échantillon a été conservé dans un sachet plastique étanche.

\section{Grand rayon $: 6 \mathrm{~m}$}




\subsection{Analyses au laboratoire}

L'extraction des nématodes s'est réalisée à partir d'aliquotes de $250 \mathrm{~cm}^{3}$ prélevées dans les échantillons de sol homogénéisés selon la technique d'élutriation (Seinhorst, 1962). Le dénombrement des nématodes s'est fait à partir de $5 \mathrm{~cm}^{3}$ de prélèvement sur les $50 \mathrm{~cm}^{3}$ de solution de nématodes obtenue après extraction. Ensuite, le nombre total de nématodes a été ramené au litre de sol.

\subsection{Analyses statistiques}

L'abondance des nématodes a été appréciée dans chaque habitat à travers la fréquence $(f)$ et la densité moyenne $(N)$. La fréquence correspond au rapport du nombre d'échantillons dans lesquels un nématode est rencontré par le nombre d'échantillons total. La fréquence ne prend pas en compte le nombre d'individus rencontrés dans l'échantillon. Les densités ont été normalisées par transformation logarithmique pour réduire les fortes variations obtenues afin de faire les analyses de variances sur les densités obtenues. La formule utilisée pour la transformation est $A i=\log [N i+1]$ où $N i=$ densité brute et $A i=$ densité normalisée.

La diversité et l'équitabilité des peuplements dans chaque type d'utilisation de sol ont été calculées respectivement à partir de l'indice de Shannon Weaver $\left(H_{S}\right)$ et de l'indice de Pielou $(J)$.

$$
\begin{aligned}
& H @ 0 \hat{i} \hat{i} 1 \\
& J @ \frac{H}{H_{\max }} ; P_{i}, \log _{2} P_{i}
\end{aligned}
$$

où $P_{i}$ : abondance relative du genre i et $S:$ nombre total de genres recensés.

L'indice d'équitabilité est utile pour comparer les dominances potentielles entre différents milieux (Pielou, 1977).

La comparaison de l'abondance (densité normalisée) et de la diversité des nématodes entre les habitats à l'échelle du paysage a été réalisée à l'aide d'analyses de variance (ANOVA) à un facteur au seuil de $5 \%$. Chaque test d'ANOVA significatif, suivi du test de Duncan, a permis de regrouper les habitats en milieux homogènes. Les relations entre les paramètres mesurés et l'indice d'utilisation de sol ont été analysées par des régressions linéaires simples au seuil de $5 \%$. Tous ces tests ont été réalisés à l'aide du logiciel STATISTICA 5.0.

\section{Résultats}

Le peuplement de nématodes est dominé en général par les nématodes libres qui représentent $68 \%$ de la nématofaune globale à l'échelle du paysage (Fig. 2). Toutefois, leur densité totale n'est pas sensible à la perturbation des habitats (Fig. 3). Le peuplement de nématodes phytoparasites, qui ne représente que $32 \%$ de la nématofaune global, est composé de neuf genres: Criconemella, Helicotylenchus, Heterodera, Hoplolaimus, Meloidogyne, Pratylenchus, Radopholus, Rotylenchulus et Xiphinema. Ce peuplement est dominé par Meloidogyne $s p$ qui représente $68,20 \%$ de la nématofaune phytoparasite (Fig. 2). Ce nématode est fréquent $(f>50 \%)$ dans tous les habitats (Tableau 1). II n'est pas sensible au type d'habitat $(F=1,43, P=0,22)$. II est suivi par Rotylenchulus $s p$ qui représente $15,60 \%$ de la nématofaune phytoparasite à l'échelle du paysage (Fig. 2). Rotylenchulus sp et Xiphinema $s p-1,60 \%$ de la nématofaune phytoparasite sont plus abondants dans les milieux forestiers (Tableau 1). Leurs densités baissent des forêts vers le domaine rural et sont significativement corrélées (Rotylenchulus $s p: r=-0,74 ; P=0,03$ et Xiphinema $s p: r=-0,77 ; P=0,03$ ) avec l'indice d'utilisation de sol (Fig. 4). De plus, I'ANOVA a mis en évidence un effet significatif $(F=2,92 ; P=$ $0,01)$ du type d'habitat sur les populations de Rotylenchulus $\mathrm{sp}$ en particulier.

Les nématodes Criconemella sp, Hoplolaimus $s p$ et Radopholus $s p$ sont rares dans tous les habitats (Tableau 1). Ils représentent moins de $1 \%$ de la nématofaune phytoparasite à l'échelle du paysage (Fig. 2). Le type d'habitat ne semble pas avoir d'effet significatif sur Helicotylenchus $s p(7,10 \%)$, Heterodera sp $(4,8 \%)$ et Pratylenchus $s p(1,7 \%)$; leurs densités ne sont pas corrélées avec l'indice d'utilisation de sol.

L'ANOVA appliquée aux indices de diversité de Shannon et d'équitabilité de Pielou montre que le type d'habitat a un effet très significatif sur la diversité $(F=4,77 ; P<0,01)$ et sur l'équitabilité $(F=6,02 ; P<0,01)$. Les milieux boisés (forêts, reboisements plurispécifiques, plantations de cacaoyers et de teck de 10 ans) ont les indices de diversité et d'équitabilité les plus élevés $(H>$ $0,74 ; J>0,74)$ par rapport aux milieux herbacés fortement perturbés $(H<0,46 ; J<0,45)$ (Tableau 2). 
Tableau 2 : Indices de diversité de Shannon et d'équitabilité de Pielou obtenus dans les différents habitats. Pour chaque indice, les valeurs portant des lettres différentes sont très significativement différentes $(P<0,01)$

\begin{tabular}{lcc}
\hline Types d'habitats & $\begin{array}{c}\text { Indice de Shannon } \\
\text { standard }\end{array}$ & $\begin{array}{c}\text { Indice de Pielou } \\
\text { standard }\end{array}$ \\
\hline Forêt primaire & $0,87 \mathrm{ab} \pm 0,17$ & $0,74 \mathrm{ab} \pm 0,11$ \\
Forêt secondaire & $1,19 \mathrm{a} \pm 0,26$ & $0,78 \mathrm{ab} \pm 0,11$ \\
Reboisements plurispécifiques & $0,98 \mathrm{a} \pm 0,19$ & $0,75 \mathrm{ab} \pm 0,10$ \\
Plantations de teck de dix ans & $0,75 \mathrm{ab} \pm 0,11$ & $0,75 \mathrm{ab} \pm 0,11$ \\
Plantations de teck de quatre ans & $0,25 \mathrm{~d} \pm 0,18$ & $0,19 \mathrm{c} \pm 0,12$ \\
Plantations de cacaoyers & $1,03 \mathrm{a} \pm 0,11$ & $0,94 \mathrm{a} \pm 0,05$ \\
Jachères & $0,33 \mathrm{~cd} \pm 0,15$ & $0,28 \mathrm{c} \pm 0,15$ \\
Milieux de cultures vivrières & $0,45 \mathrm{bcd} \pm 0,18$ & $0,45 \mathrm{bc} \pm 0,18$ \\
\hline
\end{tabular}

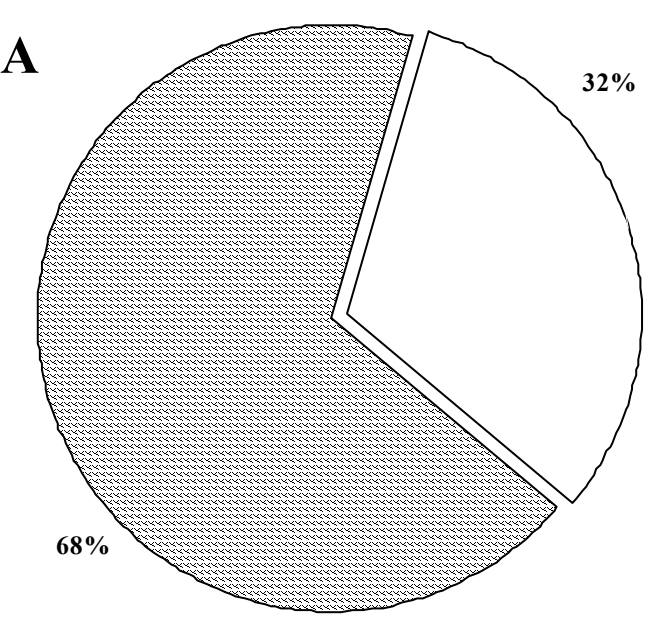

图 Nématodes libres $\square$ Nématodes phytoparasites

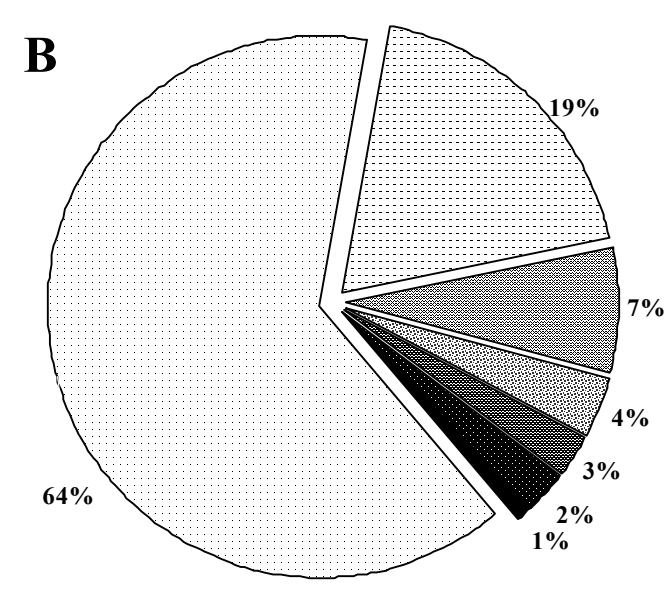

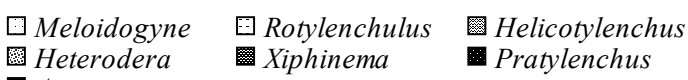
autres

Figure 2 : Abondances relatives des différentes composantes de la nématofaune à l'échelle du paysage. A : Abondances relatives des groupes trophiques par rapport à la nématofaune globale ; B : Abondances relatives des différentes populations au sein du peuplement de nématodes phytoparasites. 


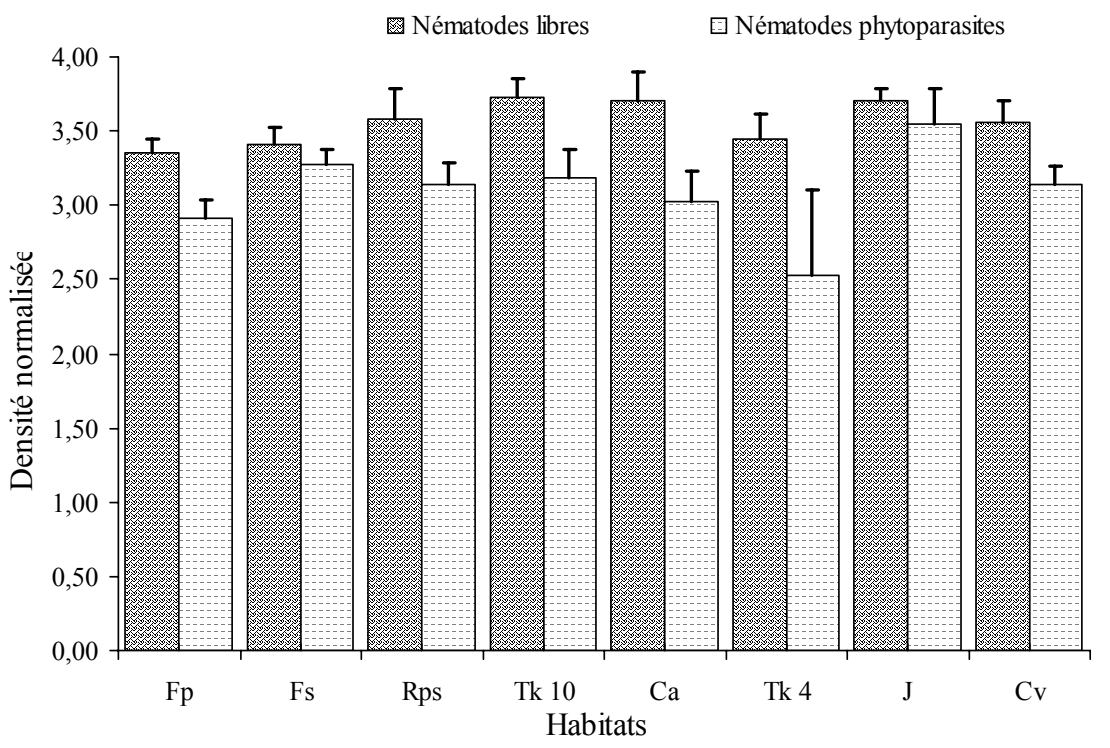

Figure 3 : Densités normalisées des nématodes libres et phytoparasites obtenues dans les différents d'habitat $(P>$ 0,05). Fp : forêt primaire; Fs : forêt secondaire ; Rps : reboisements plurispécifiques ; Tk10 : plantations de teck de 10 ans ; $\mathrm{Ca}$ : plantations de cacaoyers ; Tk4 : plantations de teck de quatre ans ; $\mathrm{J}$ : jachères et $\mathrm{Cv}$ : milieux de cultures vivrières

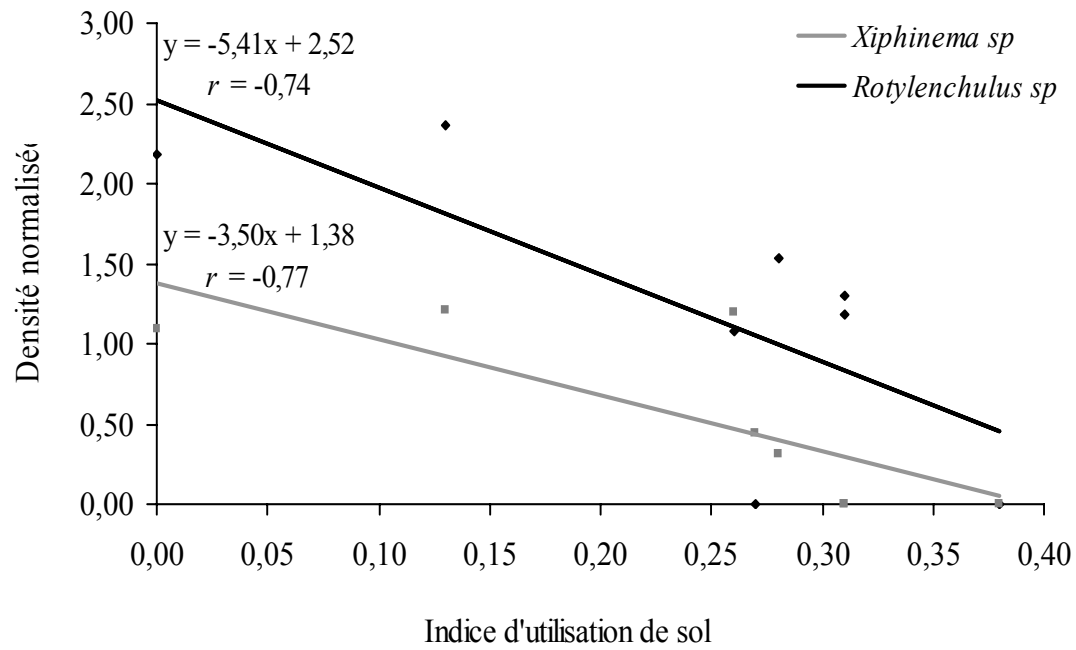

Figure 4 : Corrélations entre les populations de Rotylenchulus sp et de Xiphinema sp avec l'indice d'utilisation de sol $(P=0,03)$ 


\section{Discussion}

Quels que soient les habitats considérés, les nématodes libres dominent la nématofaune globale. Ils sont constitués de plusieurs groupes trophiques à savoir les bactériophages, les mycophages, les prédateurs et les omnivores. La densité totale des nématodes libres ne peut pas être utilisée comme indicateur de perturbations d' habitats. Néanmoins, la diversité de ce groupe de nématodes pourrait révéler leur utilité comme indicateur de perturbation de forêts (Bongers, 1990). Les omnivores en particulier ont été reconnus comme des indicateurs de milieux perturbés (Freckman \& Caswell, 1985).

Les nématodes phytoparasites présentent des réponses variables selon le type d'habitat. Meloidogyne $s p$ est fréquent dans tous les habitats. La prévalence de ce nématode phytoparasite pourrait s'expliquer par sa polyphagie. En effet, sur 241 espèces végétales d'Afrique de l'Ouest étudiées par Luc \& De Guiran (1960), ce genre a été associé à 183 plantes, soit $76 \%$ des espèces végétales présentes. II a été aussi signalé dans les zones sahéliennes (Sénégal) où il est associé aux racines d'arbres tel que le baobab (Taylor et al, 1978); ce qui indique que ce nématode est non seulement polyphage mais aussi ubiquiste. II serait intéressant d'identifier ce nématode au niveau de l'espèce afin de mieux apprécier ses propriétés écologiques. C'est également le cas de Rotylenchulus $s p$ et de Xiphinema $s p$ qui à l'inverse de Meloidogyne sp se développent mieux dans les milieux forestiers et peu perturbés.

Les espèces telles que Rotylenchulus intermedius et Xiphinema yapoense avaient déjà été découvertes en Côte d'Ivoire dans les forêts de Taï (Fortuner \& Couturier, 1983). La présence de ces deux nématodes phytoparasites dans les forêts d'Oumé au cours de la présente étude corrobore ces résultats. Xiphinema sp est absent dans les milieux fortement perturbés alors qu'il est fréquent ( $f$ e» $50 \%$ ) dans les milieux peu perturbés. Sa sensibilité à la perturbation de la forêt est marquée par la corrélation significative entre la densité et l'indice d'utilisation de sol. Ce constat est en conformité avec les études antérieures indiquant que Xiphinema $s p$ regroupe des "espèces persistantes», c'est-àdire des espèces qui se développent dans les milieux non perturbés (Bongers, 1990 ; Yeates et al., 1993 ; Bongers \& Bongers, 1998 ; Bongers, 1999). Rotylenchulus $\mathrm{sp}$ est sensible au type d'habitat. En effet, presque absent dans les milieux de cultures vivrières, ce nématode apparaît dans les jachères, avec une abondance relativement importante dans les zones reboisées et est dominant dans les plantations de cacaoyers et dans les forêts, milieux qui présentent une bonne canopée. Cette évolution indique en outre sa sensibilité à la perturbation des forêts $(r=-0,74 ; P=0,03)$. La mise en culture des sols réduit les populations de Rotylenchulus $\mathrm{sp}$. Ce résultat confirme ceux de Gnonhouri \& Adiko (2000) qui ont observé une baisse de ses populations après l'abattage et la mise en culture des jachères dans la région d'Oumé. La sensibilité de Rotylenchulus $s p$ et de Xiphinema $s p$ confirme l'utilisation des nématodes comme bioindicateurs des écosystèmes (Ettema \& Bongers, 1993 ; Freckman \& Ettema, 1993 ; Neher \& Campbell, 1994).

Les changements au niveau de la composition se reflètent dans les variations des valeurs de l'indice de diversité de Shannon. Les plus importantes valeurs sont obtenues dans les milieux protégés. La protection des forêts contre les pratiques agricoles favorise au cours du temps l'établissement d'une diversité des peuplements vivant dans ces milieux (Wardle et al., 1995). Dans le domaine rural, l'usage récurrent des sols pourrait avoir entraîné la perte de diversité liée à la dominance d'un nombre réduit de nématodes; dominance caractérisée par la faible équitabilité. Les cultures vivrières, hôtes spécifiques de certains nématodes pourraient être à l'origine de la multiplication des seuls nématodes inféodés à ces cultures.

\section{Conclusion}

La perturbation des forêts a induit des réponses variables chez les nématodes et a entraîné des modifications dans la composition des peuplements. Ces changements se traduisent par une perte de diversité due aux activités humaines. Rotylenchulus $s p$ et Xiphinema $s p$ dont les populations sont négativement corrélées à l'indice d'utilisation de sol pourraient être utilisés comme bioindicateurs pour suivre l'état de dégradation des forêts en Côte d'Ivoire. 


\section{Remerciements}

Cette étude a été réalisée dans le cadre de la mise en œuvre du projet $N^{\circ} \mathrm{GF} / 2715$ intitulé «Conservation et Gestion Durable de la Diversité Biologique dans les Sols» (CSM-BGBD). Les auteurs expriment leur gratitude à TSBF Institute of CIAT, agence d'exécution, pour la coordination des activités ainsi que la SODEFOR pour avoir permis l'accès du site d'étude.

\section{Références citées}

Bongers T., 1990. The maturity index: an ecological measure of environmental disturbance based on nematodes species composition. Oecologia 83: $14-19$.

Bongers T., 1999. The maturity index, the evolution of nematode-history traits, adaptative radiation, and cp-scaling. Plant Soil 212: 13-22.

Bongers T. \& Bongers M., 1998. Functional diversity of nematodes. Appl. Soil. Ecol. 10: 239-251.

Ettema C.H. \& Bongers T., 1993. Characterization of nematodes: colonization and succession in disturbed soil using the Maturity index. Biol. Fert. Soils 16: 79-85.

Fortuner R. \& Couturier G., 1983. Les nématodes parasites de plantes de la forêt de Taï (Côte d'Ivoire). Rev. Nématol. 6: 3-10.

Freckman, D.W. et Caswell, E.P., 1985. The ecology of nematodes in agrosystems. Annu. Rev. Phytopathol. 23: 275-296.

Freckman D.W. \& Ettema H.C., 1993. Assessing nematode communities in agrosystems of various human interventions. Agr. Ecosyst. Environ. 45: 239-261.

Gnonhouri G.P. \& Adiko A., 2000. Effets des cultures après jachère améliorée sur les populations des nématodes en Côte d'lvoire. In: Floret C., Pontanier R. \& John L.E., Eds. La jachère en Afrique tropicale (Session III). Paris, France, pp. 277-282.

Gupta V.V.S.R., 1994. The impact of soil and crop management practices on the dynamics of soil microfauna and mesofauna. In: Pankhurst C.E., Doube, B.M., Gupta. V.V.S.R., Grace P.R.R., Eds. Soil biota: Management in sustainable farming systems. Melbourne, Australie: CSIRO, pp. 107-124.
Halffter G., 1998. A strategy for measuring landscape biodiversity. Biol. Internat. 36: 3-17.

Lal R., 1991. Soil conservation and biodiversity. In: Hawskworth, D.L., Eds. The biodiversity of microorganisms and invertebrates: its roles in sustainable agriculture. Wallingford, USA: CAB International, pp. 73-81.

Luc M. \& De Guiran G., 1960. Les nématodes associés aux plantes de l'Ouest Africain. Liste préliminaire. Agron. Trop. 15: 434-449.

N'Doumé C. ; Gnessougou N. ; Tondoh J.E. et Tano Y., 2005. Selection of a benchmark research area in the agroecosystems of Central-West Côte d'Ivoire: land-use typology and mapping of the study site. Mid-term report, CSM-BGBD project, Phase 1: 1-12.

Neher D.A. \& Campbell C.L., 1994. Nematodes communities and microbial biomass in soil with annual and perennial crops. Appl. Soil. Ecol. 1: 17-28.

Pielou P.C., 1977. Mathematical ecology. New York, USA: Wiley. 159 pp.

Seinhorst J.W., 1962. Modifications of the elutriation method for extraction nematodes from soil. Nematologica 8: 117-128.

Solhenius B., Boström S. \& Sandor A., 1987. Longterm dynamics of nematodes communities in arable soil under four cropping systems. J. Appl. Ecol. 24: 131-144.

Taylor D.P., Netsher C. \& Germany G., 1978. Adansonia digitala (Baobab) a new discovered host for Meloidogyne sp and Rotylenchus reniformis: agricultural implications. Plant. Dis. Rep. 62: 276-277.

Wardle D.A., Yeates G.W., Watson R.N., \& Nicolson K.S., 1995. The detritus food-web and the diversity of soil fauna as indicators of disturbance regime in agro-ecosystems. Plant Soil 170: 35-43.

Yeates G.W., 1994. Modification and qualification of the nematode maturity index. Pedobiologia 38: 97-101.

Yeates G.W., Bongers T., De Goede R.G.M., Freckman D.W. \& Georgieva S.S., 1993. Feeding habits in soil nematode families and genera - An outline for soil ecologists. J. Nematol. 25: 315-331. 